ISSN 1981-416X

Licenciado sob uma Licença Creative Commons

\title{
Les directeurs d'école primaire face aux situations qu'ils jugent complexes: une approche compréhensive des stratégies doubles
}

\author{
Primary school principals face situations that they find \\ complex: a comprehensive approach to dual strategies
}

Los directores de escuela primaria frente a las situaciones que consideran complejas: un enfoque comprensivo de las estrategias dobles

\section{Laurence Villiers-Bouthéon*}

\section{Résumé}

Nous nous intéressons à la manière dont les directeurs d'école primaire font face aux situations qu'ils jugent complexes. Plus particulièrement, nous portons notre attention sur ce que dit une directrice d'école de Polynésie française de cette situation au moyen d'une enquête par entretien. Le cadre interprétatif construit est fondé sur Le Paradoxe et le système de Barel (1989) qui donne accès aux représentations de l'individu. La situation jugée complexe appréhendée en tant que système, est étudiée à travers la contrainte convoquée par l'agir d'un Autre laquelle s'oppose à la contrainte induite par l'une des attributions institutionnelles de la directrice. Cette double contrainte établit les niveaux logiques de la situation (le système) et constitue des infra-systèmes qui entretiennent des relations de

"LVB: Doutora em Ciências da Educação, e-mail: comm@uco.fr 
fusion-séparation. Alors, les contraintes en opposition délivrent un sens contradictoire et, la directrice perçoit le paradoxe de la situation. Pour maîtriser le paradoxe, elle met en œuvre une stratégie double par la triangulation des relations qui permet de saisir le processus de production de sens. Les combinaisons entre acteurs engagées par des tiers sujet/objet procèdent par actualisation/potentialisation de relations et forment une stratégie paradoxale de compartimentage. Ainsi, la directrice d'école maîtrise la situation jugée complexe par un jeu de visibilisation/invisibilisation des relations pour faire "comme si" le paradoxe n'existait pas.

Mots-clés: Complexe. Paradoxe. Stratégie double. Compartimentage. Triangulation. Actualisation. Potentialisation.

\section{Abstract}

We are interested in how primary school principals deal with situations they consider complex. In particular, we focus on what a school principal in French Polynesia says about this situation through a survey interview. The constructed interpretative framework is based on The Paradox and the System of Barel (1989) which gives access to the representations of the individual. The situation considered complex as apprehended as a system, is studied through the constraint summoned by the action of Another which opposes the constraint induced by one of the institutional attributions of the director. This double constraint establishes the logical levels of the situation (the system) and constitutes infra-systems that maintain fusion-separation relations. Then the constraints in opposition deliver a contradictory meaning and the primary school principal perceives the paradox of the situation. To master the paradox, it implements a double strategy through the triangulation of relations that allows us to grasp the process of meaning production. The combinations between actors engaged by third parties subject / object proceed by actualization / potentiation of relations and form a paradoxical strategy of compartmentalisation. Thus, the school director masters the situation deemed complex by a game of visibilization / invisibilization of relations to make "as if" the paradox did not exist.

Keywords: Complex. Paradox. Double strategy. Compartmentalization. Triangulation. Actualization. Potentiation. 


\section{Resumen}

Nos interesamos por la manera en la que los directores de escuela primaria hacen frente a las situaciones que consideran complejas. Más particularmente, llevamos nuestra atención en lo que dice una directora de escuela de Polinesia francesa de esta situación por medio de una investigación por conversación. El marco interpretativo construido es fundado sobre La Paradoja y el sistema de Barel (1989) que da acceso a las representaciones del individuo. La situación juzgada compleja considerado como sistema, es estudiado a través de la limitación convocada por actuarle de Otro la cual se opone a la limitación inducida por una de las atribuciones institucionales de la directora. Esta limitación doble establece los niveles lógicos de la situación (el sistema) y constituye infra-sistemas que tienen relaciones de fusión-separación. Entonces, las limitaciones en oposición libran un significado contradictorio y, la directora percibe la paradoja de la situación. Para dominar la paradoja, pone en ejecución una estrategia doble por la triangulación de las relaciones que permite coger el proceso de producción de significado. Las combinaciones entre actores involucrados por terceros sujeto / objeto proceden por actualización / potenciación de relaciones y forman una estrategia paradójica de división en compartimientos. Por lo tanto, el director de la escuela domina la situación considerada compleja por un juego de visibilización / invisibilización de las relaciones para hacer que "como si»la paradoja no existiera.

Palabras clave: Complejo. Paradoja. Estrategia dobla. División en compartimientos. Triangulación. Actualización. Potenciación.

\section{Introduction}

En Polynésie française, le directeur d'école primaire joue un rôle central en sa qualité de relais opérationnel de la politique éducative: au plus près du terrain, il évolue en relation avec des partenaires tels que les enseignants, les parents, le maire, l'inspecteur de l'éducation nationale. Nous nous intéressons aux situations que le directeur d'école primaire juge complexes, selon sa vision du monde, pour comprendre la manière dont 
il y fait face. Pour initier le raisonnement, nous définissons la complexité par ses caractéristiques: "des éléments qui entretiennent des rapports nombreux, diversifiés, difficiles à saisir par liesprit, et présentant souvent des aspects différents." (www. cnrtl.fr).

La complexité selon Barel (1989) met en exergue les liens épistémologiques entre le paradoxe et le système, à partir d'un processus de fusion-séparation des niveaux logiques. Ainsi, la complexité n'est pas un état mais la manifestation d'une dynamique cognitive de production de sens. Mais de quel sens parlons-nous? Le sens est issu d'une transformation d'un fait brut en produit "intellectuel et affectif." (p. 249). L'individu a une relation intime et sociale à la situation à travers laquelle il construit un sens subjectif. Alors, le processus de transformation évoqué permet d'accéder à une forme de "non-sens" de la situation puisque pour tout système vivant, sa "logique est illogique ou paradoxale" (1989, p. 31). Barel (AMIOT; BILLIARD; BRAMS, 1993) définit la complexité et le sens en ces termes:

Après tout, je ne suis pas sûr que la complexité ne soit pas le nom poli, un tantinet euphorisant, que se donne la paradoxalité du monde. Je fais semblant ici de vous parler de complexité et du sens, mais vous n'êtes pas dupes: c'est du paradoxe et du non-sens que nous nous entretenons (BAREL, 1989, p. 204).

Chercher à comprendre comment fait le directeur d'école pour faire face à la situation qu'il juge complexe revient à s'intéresser à la manière dont la production de sens fait face au non-sens la situation paradoxale. Autrement dit, notre objet de recherche porte sur le processus de production de sens mis en œuvre à partir du sens paradoxal de la situation jugée complexe. Pour l'auteur, l'individu peut produire un sens qui maîtrise le paradoxe, par une stratégie adaptative: une stratégie double.

En effet, la stratégie paradoxale ou stratégie double produit un sens de sorte que les logiques qui apparaissent contradictoires à un niveau fusionné puissent coexister. Elle diffère selon la manière dont elle 
intègre l'espace et le temps et comporte trois figures: le compromis, le compartimentage et le double bind.

Pour dévoiler la stratégie double mise en œuvre pour faire face à une situation jugée complexe, nous proposons l'analyse du discours d'un directeur d'école. Dans notre exemple, le cadre interprétatif construit pour son étude révèle une stratégie de compartimentage.

\section{Le processus de production de sens}

Le sens est créé par l'individu appréhendé comme un instrument fonctionnant. En l'absence de distance entre l'instrument et la fonction, l'individu est un champ perceptif qui dit sa perception (BAREL, 1989, p. 168). Autrement dit, l'individu et le terrain sur lequel il agit sont confondus ou encore, ce qui agit et ce qui est agi ne sont pas séparés. Pour saisir le concept sous-jacent de cette acception, il nous faut retenir le terme de "champ" qui met en exergue un point essentiel de la pensée de Barel: il n'existe pas deux formes d'existence, l'une sous forme d'élément et l'autre de système. L'individu n'est pas élément d'un système, il est système. Dans le prolongement, il est système d'un système, lui-même système d'un autre système. Alors, l'auteur utilise le terme de champ pour donner

une représentation de la réalité comme quelque chose d'à la fois continu et discret, en sa totalité comme en chacune de ses parties. Un champ n'est pas fait d'entités qui entrent en rapport; c'est un ensemble de flux qui se retournent sur eux-mêmes, se courbent et se croisent (se superposent) (BAREL, 1989, p. 134).

Le flux induit l'idée selon laquelle le champ a, à la fois, une réalité discrète ou digitale (fragmentaire) et, une réalité continue qualifiée d'analogique. Appréhender l'individu comme un champ perceptif permet de concevoir que, lorsqu'il dit sa perception, il y a une émission simultanée et de la même source d'un message (une réalité digitale) et 
un méta-message (réalité analogique). Le méta-message est autoproduit: il émane du message. Ce méta-message contredit en partie le message. Il n'y a pas de message innocent, de message qui ne comportât pas de méta-message contredisant en partie le message. "Il est plus facile de faire semblant d'ignorer le côté paradoxal de toute situation, que de l'exorciser pour de vrai" (BAREL, 1989, p. 88).

Finalement, l'individu est autoréflexif puisqu'il exprime son propre sens. Et, cette autoréflexion génère autre chose qu'un message digitalisé: le méta-message ou message analogique.

Message et méta-message s'établissent en niveaux logiques ou infra-systèmes. Leur étagement construit le sens que l'individu attribue à la situation. Le sens est un système dont les infra-systèmes entrent en mouvement de fusion-séparation: là se crée le paradoxe. L'auteur décrit ce processus au travers du phénomène de hiérarchie enchevêtrée dont les niveaux sont "à la fois séparés et fusionnés", "hiérarchisés et ne le sont pas” selon l'acception suivante:

dans son épure la plus simple, un univers à deux objets, le phénomène de la hiérarchie enchevêtrée signifie ceci: chacun de ces deux objets est tour à tour le sommet et la base de la hiérarchie. Chacun prend la place de l'autre dans une oscillation qui ne peut s'arrêter (BAREL, 1989, p. 224-226).

Les stratégies simples ou d'évitement du paradoxe consistent à ignorer le paradoxe "ce qui se traduit concrètement par l'adoption d'une stratégie linéaire dans une situation qui ne l'est pas, ou d'une stratégie qui choisit l'un des termes du paradoxes, ou bien encore d'une stratégie d'abstention d'action là et quand l'action s'impose" (BAREL; MITANCHEY, 1990, p. 10).

Mais selon Barel, l'individu va chercher à maîtriser le paradoxe car les individus ne sont pas "condamnés à la passivité" (BAREL, 1989, p. 240) devant le paradoxe social. Ils peuvent le maîtriser, lui faire face afin de "rendre ce dernier plus vivable et plus supportable qu'il ne le serait si les hommes restaient passifs devant lui" (BAREL, 1989, p. 241). 
La maîtrise que l'individu peut avoir sur le paradoxe ne le fait pas disparaître: il demeure en arrière plan de la situation. Les stratégies qu'ils déploient sont "des manières de vivre avec ces paradoxes sans s'y perdre." (BAREL, 1989, p. 230). La maîtrise du paradoxe s'entend au travers de stratégies doubles selon lesquelles il devient possible de "refuser le choix en même temps qu'on l'effectue, de réaliser une exclusion inclusive, le choix sans faire le choix" (BAREL, 1989, p. 230).

Ces stratégies doubles ne sont pas conscientisées parce qu'un mécanisme de refoulement entre en jeu qui fait de la stratégie, pour son auteur, un paradoxe qui s'enfouit, qui se cache à lui-même, pour pouvoir exister comme paradoxe" (BAREL, 1989, p. 182).

La stratégie double poursuit un but unique ou dominant dont la réalisation est assortie de "la réalisation d'autres buts qui peuvent être en conflit ou en contradiction" (BAREL, 1989, p. 184). Elle n'est donc pas la juxtaposition de deux stratégies simples. Elle est stratégie paradoxale dans la mesure où elle actualise un choix sans détruire le non choix. Celuici est potentialisé. A tout moment, il peut devenir le choix principal et être actualisé: "voilà ce que signifie la maîtrise du paradoxe qui consiste à choisir en refusant de choisir." (p. 242). La stratégie double apparaît comme un jeu de décidabilité-indécidabilité, l'indécidabilité assurant la réversibilité du choix et non une simple indécision (p. 243).

Parmi les figures stratégiques qu'énonce l'auteur (compartimentage, compromis et double bind) et qui se distinguent selon leurs caractéristiques spatiale et temporelle, nous retenons la stratégie double du compartimentage. Cette stratégie intervient lorsque, pour faire face à la situation complexe, l'individu mène plusieurs stratégies qui se déploient au travers de stratégies simples et semblent indépendantes. Le paradoxe devient invisible sans que sa dissimulation ne soit pour autant le fruit d'un acte délibéré. Et, "C'est seulement à un méta-niveau que les relations apparaissent.” (BAREL, 1989, p. 198). Le fractionnement des stratégies simples engendre une rupture de l'unité de lieu: "Quand une stratégie double apparaît sous la forme de plusieurs stratégies non implicatives, il y a rupture de la règle dramatique de l'unité de temps et 
de lieu en ce sens que si ces stratégies peuvent à l'occasion se dérouler simultanément, l'unité de "lieu" se trouve violée".

On peut alors s'interroger sur la manière dont la stratégie double se déploie. Les stratégies paradoxales engagent un processus de triangulation qui, avec "son jeu sur le visible et l'invisible" (BAREL, 1982 p. 337), établit la connivence secrète des stratégies simples qui composent la stratégie double (p. 268).

Les stratégies paradoxales font intervenir des tiers (sujet et/ou objet) qui évitent la confrontation directe des contradictions incarnées par les acteurs de la situation.

Le phénomène de triangulation engage "des relations sans relation" (BAREL, 1987, p. 261) sachant que la relation est définie comme "un entre-deux, à la fois interne et externe aux "objets" reliés, et particulièrement sujette, de ce fait, à l'invisibilisation” (p. 21).

Alors, lorsque le tiers établit une relation visible avec un acteur, il permet ainsi de voir ce qui rend invisible la relation invisibilisée par le tiers. La visibilisation et l'invisibilisation des relations correspondent à leur actualisation et potentialisation.

Autrement dit, pour produire un sens qui maîtrise le paradoxe, la stratégie double opère par la triangulation laquelle engage un tiers objet et/ou un tiers sujet dans la situation paradoxale. Des relations sont actualisées et d'autres, potentialisées. Ainsi, le sens produit procède d'un jeu sur le visible et l'invisible social.

Nous proposons d'étudier la manière dont le sens est produit par une stratégie de compartimentage à partir de l'analyse du discours d'une directrice d'école ayant trait à une situation qui à ses yeux, est complexe. Pour ce faire, nous utilisons un cadre interprétatif construit qui comporte deux phases d'étude. Une première phase met à jour le sens paradoxal de la situation en retenant l'une des attributions institutionnelles de la directrice d'école. Une seconde phase porte sur l'analyse de la stratégie double afin de comprendre le processus de production de sens qui permet au directeur de faire face au paradoxe. Il s'agit alors d'identifier la stratégie 
double et la manière dont le processus de triangulation se saisit du jeu de l'actualisation/potentialisation pour produite un sens.

\section{Analyse de donnees}

Nous partons à la rencontre des représentations que la directrice d'école se fait des situations professionnelles jugées complexes en utilisant comme instrument de recueil des données l'enquête par entretien.

La directrice d'école interviewée exerce dans une île éloignée de la capitale de la Polynésie française que constitue Tahiti. Dans son discours, elle évoque une difficulté relationnelle avec le partenaire communal, découverte par la directrice lors d'une réunion de rentrée à laquelle participaient notamment les enseignants et les parents. Devant l'auditoire, le maire affirme que l'école ne peut remplir ses missions en raison de difficultés relationnelles internes.

je ne m'attendais pas du tout euh à cette réaction et donc il intervient pour dire que ben: "Voilà ma position euh, je pense qu'il y aura des difficultés parce que au sein de cette école, ils ont des difficultés relationnelles euh au niveau de leur équipe elle-même et donc, l'école, ça ne peut pas marcher".

Sur le moment, la directrice n'intervient pas par convenance. Plus tard, elle cherchera à rencontrer le maire sans pour autant y parvenir.

Et donc, je l'ai relancé en demandant à ce qu'on se revoit et qu'on discute un peu de tout cela largement, largement. Et donc, je comprenais pas cette situation et euh, j'avais du mal à le rencontrer parce qu'il se déplace beaucoup [...] et donc, j'ai dû passer par l'inspectrice pour résoudre cette situation.

Alors, la directrice d'école sollicite l'inspectrice qui lui propose de communiquer sur ce que l'école fait. Elle réalise un support numérique pour "faire savoir" au maire par l'intermédiaire de l'inspectrice. 
l'inspectrice a proposé comme solution de, d'insister sur ce que l'école fait parce que en fait, la mairie ne voit pas ce que l'école fait. Elle a vu ou entendu des choses qu'elle n'aurait pas faites mais elle n'a pas à côté, retenu ou vu ou entendu ce qu'elle a fait. Et donc, elle m'a demandée avec l'équipe, de mettre en avant au travers de PowerPoint, au travers de photos, au travers de documents, ce qui avait été fait pour que, et au niveau communal et au niveau parental, ils en soient conscients.

Finalement, au cours d'une réunion communale, le maire reconnaît publiquement le travail effectué.

Et, ce soir là, il m'appelle et me demande de le rejoindre devant. Alors j'y vais, évidemment. Je m'assois. Je me demande ce que je fais devant et je me sens honorée. Je me dis: "Là, là... C'est quand même un pas vers moi, donc voilà. Est-ce que ça va faire la conciliation avec l'école que je représente?". Bon, au milieu de la réunion, il demande à la population d'applaudir l'école, de m'applaudir pour l'école, pour tout ce que l'école a fait.

En parallèle de l'intervention de l'inspectrice auprès du maire, la directrice tâche de communiquer avec les parents de l'école "pour se connaître aussi autrement." Alors, elle organise des réunions parentsenseignants sous le préau de l'école et une manifestation Halloween qui emporte un large succès.

Ensuite, euh, d'essayer euh, donc de communiquer autrement avec les parents. Et donc, du coup cette année, pour cette rentrée, j’ai proposé à l'équipe que nous euh organisions, aménagions euh le préau différemment pour la réunion parentale [...] on s'installe différemment pour monter aux parents que à ce moment là, nous sommes tous pareils, que nous sommes sur un même pied, le pied de: "Comment va-t-on faire pour qu'ensemble, on arrive à aider nos enfants à réussir?”.

Il y a une autre stratégie que nous avons mise en place [...] nous avons eu notre première sortie de nuit Halloween et c'était pendant les vacances. Et bien, on ne s'attendait pas à cela: on a regroupé 200 à 300 personnes. 
La directrice met ainsi en exergue l'importance de communiquer et de se connaître: "il semble qu'il manquait ce petit volet relationnel, vraiment, entre êtres humains pour partager des moments [hésitation] joyeux".

Les deux phases d'étude évoquées nous guident dans l'analyse en observant la situation jugée complexe avant d'identifier la stratégie double mise en œuvre.

\section{La situation jugée complexe}

Pour saisir la manière dont la directrice perçoit la situation, il convient de se placer dans un contexte d'hétéro-référence qui prend la forme de l'autoréférence indirecte. En effet, la directrice d'école parle d'autrui, et cette hétéro-référence lui permet d'énoncer ses relations (autoréférence indirecte) avec les partenaires du système éducatif dont le maire et les parents.

Ainsi, deux systèmes entrent en interaction avec celui de la directrice d'école lors de l'exposé de la situation complexe: celui du maire et celui des parents. Les actions du maire qui manifeste "sa position anti-scolaire" entrent en interaction avec les attributions attachées au rôle de la directrice d'école institutionnalisées par l'arrêté en Conseil des Ministres de Polynésie française du 24 juillet 1996 selon lequel:

Il (le directeur d'école) représente l'institution auprès de la commune. Il veille à la qualité des relations avec les partenaires du système éducatif, notamment constitués par les autorités municipales, les parents d'élèves, le monde économique et les associations socio-culturelles [...] (CONSEIL DES MINISTRES DE POLYNÉSIE FRANÇAISE, 1996, p. 3)

Dans le cas de difficultés relationnelles profondes [...] le directeur pourra se voir retirer, par l'autorité hiérarchique, la fonction de direction occupée (Art. 30-1.9). 
La directrice doit veiller à la qualité des relations qu'elle entretient avec les partenaires du système éducatif dont le maire. Or, ces relations sont dégradées. Pour rétablir la qualité des relations, elle doit rencontrer le maire. Mais, le maire ne la reçoit pas.

La situation d'interaction se formule ainsi: la directrice d'école doit veiller à la qualité des relations avec le maire sans veiller à la qualité des relations, puisqu'elle ne le peut pas. Comme le maire ne la reçoit pas, le problème semble insoluble. La situation exposée par la directrice est paradoxale.

On sait, de la façon la plus simple et la plus authentique, qu'on a affaire à un paradoxe, chaque fois que l'on rencontre une situation dans laquelle il est nécessaire de faire, de dire ou de penser une chose et le contraire de cette chose. On peut dire aussi que le paradoxe s'exprime dans la double obligation de choisir et de ne pas choisir entre deux ou plusieurs solutions à un problème donné. Ou bien, qu'il est un problème insoluble ayant une solution apparente ou réelle; ou bien que le paradoxe est ce qui se localise dans un espace-temps déterminé tout en se montrant présent et actif dans d'autres espace-temps (BAREL, 1989, p. 223).

La directrice émet un message et simultanément, un méta-message qui comporte en partie un message contradictoire. Dans notre étude, le message est établi au regard de l'action du maire qui contrarie la réalisation d'une des attributions de la directrice d'école à savoir veiller à la qualité des relations avec les partenaires du système éducatif dont la mairie. Lorsque le message est injonctif, le méta-message oppositif possède cette même caractéristique. Alors, la directrice est sujette à une double contrainte:

la contrainte liée à son attribution: devoir veiller à la qualité de ses relations avec le maire;

la contrainte de fait, liée à l'indisponibilité du maire. Elle ne peut pas le rencontrer. Ne pas veiller à la qualité des relations ne dépend pas d'elle: "ne pas pouvoir" manifeste la contrainte subie.

Devoir et ne pas pouvoir supposent une indépendance car le devoir ne modifie pas le pouvoir; seulement, lorsqu'ils sont réunis dans 
un même message, ils acquièrent une connivence profonde et établissent une relation de contrariété. Elle doit veiller à la qualité des relations sans pouvoir y veiller, ce qui est paradoxal.

L'identification de la situation paradoxale par les ordres normatifs qui définissent le rôle du directeur d'école (ses attributions) nous conduit à formuler deux propositions (A et B) qui structurent en niveaux logiques les infra-systèmes de la situation soit:

- proposition A: énoncé 1 qui correspond à l'ordre normatif (l'attribution visée par le message);

- proposition B: énoncé 2 qui correspond à "ce qui s'oppose aux attributions".

\section{Le premier niveau logique:}

La directrice doit veiller à la qualité de ses relations avec les partenaires du système éducatif dont le maire eu égard à ses attributions.

Enoncé 1: devoir veiller à la qualité des relations.

Auto-référence indirecte 1: la directrice dans ses relations aux partenaires du système éducatif dont le maire.

\section{Le second niveau logique:}

La directrice a pour but de rencontrer le maire pour lui "faire savoir" ce que l'école fait, mais elle ne peut pas le faire car il ne la reçoit pas.

Enoncé 2: ne pas pouvoir veiller à la qualité des relations.

Auto-référence indirecte 2: la directrice dans ses relations au maire.

Les énoncés 1 et 2 "dessinent des frontières de types logiques: ils hiérarchisent et ponctuent la réalité" (BAREL, 1989, p. 49). Chacune de ces logiques sont des infra-systèmes de la situation jugée complexe (le système). Les niveaux logiques sont étagés pour former ce que Barel appelle "une hiérarchie enchevêtrée". Nous proposons alors une 
modélisation heuristique du processus paradoxal adaptée de l'illustration donnée par Gouraud (2011, p. 60).

Figure 1 - Représentation heuristique: les niveaux logiques

Niveau 1: Dans ses relations aux partenaires du système éducatif dont le maire

A: Devoir veiller à la qualité des relations.

Non A: Ne pas devoir veiller à la qualité des relations.

Niveau 2: Dans ses relations au maire

B: Ne pas pouvoir veiller à la qualité des relations.

Non B: Pouvoir veiller à la qualité des relations.

Font : De l'Auteure.

C'est au moment où les niveaux 1 et 2 fusionnent, termes à termes, que les propositions, délivrent un sens contradictoire: la directrice doit rencontrer le maire (A) mais elle ne peut pas le faire (B).

\section{Le dévoilement de la stratégie double}

La stratégie double permet la coexistence des niveaux logiques contradictoires en redéfinissant les interactions par le processus de triangulation qui convoque un tiers sujet et/ou objet. Cette redistribution relationnelle prend plusieurs formes (le compartimentage, le compromis, le double bind) et produit un sens: des niveaux logiques sont ainsi surimposés aux niveaux logiques contradictoires. Le paradoxe n'a pas disparu mais la création de sens permet de faire "comme si” le paradoxe n'existait pas. 


\section{La stratégie de compartimentage révélée par l’analyse:}

La stratégie double suppose un but unique ou dominant qui, dans notre étude, est de veiller à la qualité des relations avec le maire et la fusion paradoxale de deux stratégies simples: l'une visant à "faire savoir" au maire par des supports matériels (stratégie 1) et l'autre à communiquer autrement avec les parents soit "faire savoir" aux parents (stratégie 2).

Pour identifier la stratégie double, nous en venons à ses caractéristiques spatiale et temporelle. La stratégie paradoxale à l'œuvre concerne:

- plusieurs espaces: l'école, lieu où se construisent les relations avec les parents que ce soit pour les réunir sous le préau ou pour servir de point de regroupement au départ et à l'arrivée de la manifestation Halloween et, le lieu des échanges avec le maire à la mairie;

- un moment qui s'établit sur deux années. Les stratégies simples se déroulent successivement, à l'échelle du temps de la situation, et simulent leur indépendance dans le sens où elles sont non implicatives.

Pour maîtriser le paradoxe, la directrice compartimente ses stratégies. La stratégie double de compartimentage retient le but dominant qui est de veiller à la qualité des relations avec les partenaires du système éducatif dont le maire, conformément à ses attributions. Elle produit des choix qui s'ignorent: l'un visant à "faire savoir" au maire par des supports matériels et par l'intermédiaire de l'inspectrice (stratégie simple 1); l'autre consiste à communiquer autrement avec les parents au travers de diverses actions (stratégie simple 2): des réunions parents-enseignants, la manifestation Halloween. Ces stratégies simples se déploient sur des lieux et auprès d'acteurs différents. Elles simulent ainsi leur indépendance. La stratégie double déployée est celle du compartimentage. 


\section{Le processus de la triangulation}

Le processus de la triangulation permet de faire coexister des contradictions ou des contraires en dissimulant le paradoxe par une/des relations triangulaires.

Le "faire savoir par un intermédiaire" au maire (stratégie simple 1) détermine l'acceptation de la directrice par le maire. Pour maîtriser le paradoxe, la directrice procède à la triangulation de la relation duale. Pour la stratégie simple 1 , la directrice d'école sollicite un tiers sujet: l'inspectrice. Il s'agit de "demander l'appui de notre inspectrice pour m'aider à résoudre cette situation"; "j'avais du mal à le rencontrer parce qu'il se déplace beaucoup. [...] et donc, j'ai dû passer par l'inspectrice pour résoudre cette situation.". L'inspectrice est le tiers-sujet chargé de médier la relation entre la directrice d'école et le maire.

Et, la directrice mobilise un tiers-objet à la demande de l'inspectrice: un document numérique (PowerPoint) pour faire savoir ce que l'école fait.

Ainsi, le tiers sujet "inspectrice" et le tiers objet "document numérique PowerPoint" interviennent pour mener la stratégie simple 1 par la triangulation de la relation duale directrice-maire.

Pour mener la stratégie simple 2 qui vise à "faire savoir personnellement" aux parents, la directrice d'école renforce la qualité de ses relations avec les parents par des tiers objets. Elle met en oeuvre diverses actions "pour communiquer autrement avec les parents" en organisant une réunion parents-enseignants et une manifestation Halloween.

Ainsi, les tiers objets "réunion parents-enseignants" et "manifestation Halloween" interviennent pour mener la stratégie 2 par la triangulation de la relation duale directrice-parents.

Le jeu des actualisations et potentialisations des relations entre les acteurs engagés dans la situation (acteurs de l'autoréférence et de l'hétéro-référence) est observé selon la figure stratégique mise en œuvre. Nous ne cherchons pas à représenter ce processus pour en dégager un mode opératoire. Mais, nous nous attachons à percevoir le jeu du visible 
et de l'invisible par le processus de triangulation afin d'appréhender le phénomène de production de sens de la stratégie double en retenant ses principales caractéristiques. Il s'agit de saisir les interactions selon un autre mode d'existence de la réalité sociale:

Il y a sans doute plusieurs modes d'existence de la "réalité" sociale, et notamment un mode d'existence comme potentialité que l'on a tendance à confondre avec l'inexistence, parce que l'on est obnubilé par le seul potentiel qui parvient à s'actualiser (BAREL, 1982, p. 13).

Pour la stratégie de compartimentage, l'indépendance des stratégies doubles est simulée par l'invisibilisation d'une relation duale. Cette relation duale est construite sur un non événement, soit un événement potentialisé, créant une connivence entre ses termes: "Mais on n’observe pas assez qu'un événement n'arrive jamais seul, mais toujours accompagné d'un non-événement." (BAREL, 1982, p. 13). Il est rendu invisible par des tiers distincts aux deux niveaux stratégiques ou des tiers dont le caractère commun est ignoré en raison du déploiement des stratégies simples en des lieux différents. Il s'agit d'éviter tout point de contact entre les stratégies.

Figure 2 - Stratégie de compartimentage: actualisation, potentialisation des relations

\begin{tabular}{|c|c|c|c|}
\hline \multirow{2}{*}{$\begin{array}{l}\text { Intitulé des } \\
\text { infra-systèmes } \\
\text { Buts }\end{array}$} & Auto-référence & Hétéro-référence & \multirow{2}{*}{$\begin{array}{c}\text { Identification } \\
\text { des } \\
\text { Tiers sujets / Tiers objets }\end{array}$} \\
\hline & \multicolumn{2}{|c|}{ Représentation des relations } & \\
\hline $\begin{array}{l}\text { Stratégie } 1 \\
\text { Faire savoir par } \\
\text { un intermédiaire }\end{array}$ & $\begin{array}{l}\text { Directrice } \\
\stackrel{\mathrm{A}}{\longleftrightarrow}\end{array}$ & $\uparrow^{\mathrm{C}}$ & $\begin{array}{l}\text { Tiers sujet: IEN } \\
\text { Tiers objet: document numérique } \\
\text { PowerPoint }\end{array}$ \\
\hline $\begin{array}{l}\text { Stratégie } 2 \\
\text { Faire savoir } \\
\text { personnellement }\end{array}$ & $\begin{array}{l}\text { Directrice } \\
\stackrel{\mathrm{B}}{\longrightarrow}\end{array}$ & Parents & $\begin{array}{l}\text { Tiers objets: réunion parents- } \\
\text { enseignant, manifestation Halloween }\end{array}$ \\
\hline
\end{tabular}

Font :De l'Auteure. 


\section{Invisibilisation de la relation de confrontation par un tiers sujet}

Le tiers sujet, l'Inspectrice de l'Education Nationale (IEN) évite la confrontation directe entre la directrice et le maire ("Et donc, elle m'a demandée avec l'équipe, de mettre en avant [...] ce qui avait été fait pour que au niveau communal et au niveau parental, ils en soient conscients").

Pour la stratégie simple 1 (faire savoir au maire), la triangulation qui s'établit a l'aspect d'une relation duale: l'inspectrice intervient à la place de la directrice dans les relations au maire, à la mairie. Elle mobilise un tiers objet qui introduit "un terme "neutre" dans les rapports sociaux" (BAREL, 1982, p. 346): un support numérique PowerPoint. La relation entre le maire et l'IEN est actualisée de sorte que la relation de confrontation entre la directrice et le maire soit invisibilisée [A].

Processus de fractionnement du paradoxe: Création d'une relation visible entre le directeur d'école et un Autre par un tiers objet

Dans la stratégie simple 2 (faire savoir aux parents), une relation triangulaire est construite en créant des tiers objets c'est à dire des actions de communication (la réunion parents-enseignants et la manifestation Halloween) pour faire savoir ce que l'école fait, au niveau parental. La relation entre la directrice et les parents est ainsi rendue visible [B]. La relation duale directrice d'école - parents est médiée par la réunion parentale et la manifestation Halloween. Pour mener cette stratégie 2, le lieu de regroupement est l'école.

\section{Processus d'invisibilisation de la relation entre les stratégies} simples: création d'un événement potentialisé et invisibilisation de la relation duale

Concernant l'école, les parents peuvent influencer l'opinion du maire. Il s'agit d'un événement potentialisé [C]. La stratégie 2 est mise en œuvre par rapport à cet un événement potentialisé.

Les stratégies simulent leur indépendance en mobilisant des tiers sujets/objets distincts en des lieux différents. Cette invisibilisation de la relation entre le maire et les parents est nécessaire pour que la stratégie double puisse être mise en œuvre par des stratégies simples qui semblent s'ignorer. 
La directrice fait savoir de manière différenciée ce que l'école fait: au maire, par l'intermédiaire de l'inspectrice en utilisant un support numérique PowerPoint (stratégie 1), l'espace de la mairie étant référent, et aux parents, personnellement en organisant une réunion parentale et une manifestation Halloween (stratégie 2), l'espace école servant au regroupement. Elle renforce ainsi la qualité de ses relations avec les parents ce qui peut venir influencer voire renforcer la qualité de ses relations avec le maire.

La stratégie paradoxale éclatée en stratégies simples (non paradoxales en apparence) tient son efficacité à la manière dont elles se combinent pour former la stratégie double: l'invisibilité (BAREL, 1987, p. 287). Ces stratégies simulent ainsi leur indépendance. C'est la triangulation aux deux niveaux de la réalité qui établit un rapport invisible entre les parents et le maire: "La relation ne s'établit que s'il y a motif à relation, et ce motif ne peut être uniquement la relation elle-même" (BAREL, 1987, p. 275).

\section{Conclusion}

Nous cherchons à comprendre la manière dont la directrice d'école primaire interrogée fait face à une situation vécue qu'elle juge complexe. Au regard des concepts de la systémique paradoxale de Barel (1989), nous saisissons par un cadre interprétatif construit, le processus de production de sens de la stratégie double que le directeur d'école élabore pour maîtriser le paradoxe de la situation jugée complexe.

Lorsque l'individu dit sa perception, il émet un message et un méta-message contradictoire qui établissent des niveaux logiques hiérarchisés et non-hiérarchisés. Ils entrent en mouvement en participant à processus de fusion-séparation de niveaux. Au moment de leur fusion, termes à termes, message et méta-message sont confondus; il en résulte un message paradoxal. Ainsi, le message paradoxal est issu d'un processus dans lequel les niveaux logiques sont disjoints puis conjoints, excluant chacun de leurs termes avant de les inclure de nouveau dans un 
mouvement récursif. Le processus paradoxal est un construit en soi qui, par une dialectique contemporaine "apporte(r) à la science de la cognition une ressource méthodologique originale et puissante [...] dans l'exercice du processus cognitif" (AMIOT; BILLIARD; BRAMS, 1993, p. 95).

A partir de l'analyse du système que constitue la situation, unité de base de l'analyse, nous avons observé que la directrice d'école déploie une stratégie double de compartimentage pour faire face la situation jugée complexe. Ainsi, nous accédons au processus de production de sens qui permet la maîtrise du paradoxe: le phénomène de triangulation engage des tiers sujet et/ou objet. Alors, un jeu d'actualisation/potentialisation rend visible/invisible certaines relations entre sujets/objets de la situation pour faire "comme si" le paradoxe n'existait pas.

L'exemple de situation présenté est issu d'une étude doctorale développée par l'auteure de cet article, dirigée par M. Bergier, Professeur à l'Université Catholique de l'Ouest d'Angers, qui prolonge l'analyse en s'interrogeant sur ce qui convoque les tiers sujet/objet, leur présence n'étant pas le fruit du hasard. L'interprétation du sens paradoxal de la situation et du sens produit par la triangulation s'enrichit des concepts de la théorie des économies de la grandeur (BOLTANSKI; THÉVENOT, 1991) pour comprendre la manière dont est retenu le tiers sujet/objet engagé dans le processus de production de sens aux fins de faire face au non-sens de la situation jugée complexe.

\section{Références}

AMIOT, M.; BILLIARD, I.; BRAMS, L. Système et paradoxe : Autour de la pensée d’Yves Barel. Paris: Editions du Seuil, 1993.

BAREL, Y. La Marginalité sociale. Paris: Presses Universitaires de France, 1982.

BAREL, Y. Le paradoxe et le système. Grenoble: Presses Universitaires de Grenoble, 1989. 
BAREL, Y. ; MITANCHEY, N. Paradoxe de la pédagogie, pédagogie du paradoxe, 1990. Disponible en: <http://www.intelligence-complexite.org/fileadmin/docs/1505barelparadoxe.pdf $>$. Accès dans : 23 fév. 2017.

BOLTANSKI, L.; THÉVENOT, L. De la justification: Les économies de la grandeur. Paris: Gallimard, 1991.

CONSEIL DES MINISTRES DE POLYNÉSIE FRANÇAISE, 24 juillet 1996.

GOURAUD, F. Famille(s) sous aide contrainte. Paris: L'Harmattan, 2011.

Reçu : 02/10/2018

Received: 10/02/2018

Recibido: 02/10/2018

Approuvé : 09/10/2018

Approved: 10/09/2018

Aprobado: 09/10/2018 